Recherches en histoire de l'art, histoire des civilisations, archéologie, anthropologie et muséologie

$4 \mid 2014$

Cahiers 4

\title{
Dans collection, il y a collecte...
}

\section{Dominique Jarrassé}

\section{(2) OpenEdition \\ Journals}

Édition électronique

URL : http://journals.openedition.org/cel/480

DOI : $10.4000 / \mathrm{cel} .480$

ISSN : 2262-208X

Éditeur

École du Louvre

Édition imprimée

Date de publication : 1 avril 2014

Référence électronique

Dominique Jarrassé, "Dans collection, il y a collecte... », Les Cahiers de l'École du Louvre [En ligne], 4 | 2014, mis en ligne le 01 avril 2014, consulté le 19 avril 2019. URL : http://journals.openedition.org/ cel/480; DOl : 10.4000/cel.480

\section{(c) $(1) \&$}

Les Cahiers de l'École du Louvre sont mis à disposition selon les termes de la licence Creative Commons Attribution - Pas d'Utilisation Commerciale - Pas de Modification 4.0 International. 


\section{Cahiers de l'École du Louvre}

recherches en histoire de l'art, histoire des civilisations archéologie, anthropologie et muséologie

Numéro 4. Avril 2014

Dans collection, il y a collecte...

Dominique Jarrassé

Article disponible en ligne à l'adresse :

http://www.ecoledulouvre/cahiers-de-l'ecole-du-louvre/numero4avril2014/Jarrasse.pdf

Pour citer cet article :

Dominique Jarrassé, « Dans collection, il y a collecte... », Cahiers de l'École du Louvre, recherches en histoire de l'art, histoire des civilisations, archéologie, anthropologie et muséologie [en ligne] n 4, avril 2014, p. 21 à 23.

\section{ccreative \\ ㅌ.) $\ominus$ \$}

(C) École du Louvre

Cet article est mis à disposition selon les termes de la Licence Creative Commons Attribution - Pas d'utilisation commerciale - Pas de modification 3.0 non transposé. 


\section{Cahiers de l'École du Louvre recherches en histoire de l'art, histoire des civilisations archéologie, anthropologie et muséologie \\ Numéro 4. Avril 2014}

Sommaire

Éditorial

p. 1

\section{Étude}

Le musée de Sculpture comparée au prisme de la collection de cartes postales éditées par les frères Neurdein (1904-1915)

Dominique Jarrassé et Emmanuelle Polack

p. $2-20$

Dossier:

Les modalités de la collecte : rapt, troc, marché, fouilles, don...

Et leur impact sur l'objet

Dans collection, il y a collecte...

Introduction de Dominique Jarrassé.

p. $21-23$

Entrer en collection. Pour une ethnographie des gestes et des techniques de collecte

Julien Bondaz

p. $24-32$

De la meilleure façon de constituer une collection. Le cas des émaux «byzantins » de Mikhaill Botkine

Aglaé Achechova.

Edme Antoine Durand (1768-1835) : un bâtisseur de collections

Louise Detrez p. $45-55$

Réunir une documentation pour l'Art Brut : les prospections de Dubuffet dans l'immédiat après-guerre au regard du modèle ethnographique Baptiste Brun p. $56-66$

Travailler sur des objets en faisant se confronter les points de vue : regards croisés sur le langage fou : l'exemple des écrits bruts

Vincent Capt p. $67-75$

D’un regard-pilote à l'écart. L'impact de Dubuffet sur les collectes de l'association L'Aracine

Déborah Couette. p. $76-86$

La cabane éclatée. Morcellement des objets immobiliers apparentés à l'art brut

Roberta Trapani .p. $87-95$ 


\section{Dominique Jarrassé}

Dans une journée d'études qui concluait le séminaire doctoral 2012-2013 sur la qualification de l'objet ${ }^{1}$, des chercheurs se sont réunis pour mener une réflexion sur les processus qui définissent l'objet non pas tant dans sa nature qu'à travers les mutations de son statut. Il était apparu pertinent de se pencher sur ce moment crucial de la biographie des objets qu'est la collecte : nous empruntons sciemment cette notion à l'anthropologie, plutôt qu'à l'histoire de l'art pour décaler la perception du mouvement d'appropriation. La manière dont un objet entre dans une collection, les modalités selon lesquels il perd sa fonction ou en change, les circonstances de son acquisition ou de son don ont un impact sur sa nature, non pas intrinsèque, mais déterminée par les regards différents qui lui sont portés, et sur les connotations qui s’attachent à lui ; nous avons déjà engagé la réflexion sur cette définition dynamique de nos objets dans l'introduction de ce séminaire ${ }^{2}$. Les articles ici réunis en forment une illustration qui privilégient deux champs, la construction des collections qui peut aller jusqu'à la falsification, d'une part, et le cas très spécifique de la collecte en matière d'art brut, d'autre part. Pour cette dernière partie, nous rejoignons les objectifs définis par le programme "Anthropologie et histoire des arts » du GDRI Musée du Quai Branly/CNRS qui établit ce rapprochement ${ }^{3}:$ "S'intéresser à l'entrée d'artefacts extra-occidentaux dans le monde de l'art implique évidemment de revenir sur la construction de la catégorie d'"art primitif" et d'étudier le rôle de l'esthétique "primitiviste" dans le regard occidental sur les arts exotiques. Si la question de l'artification se pose pour les objets ethnographiques consacrés en arts des civilisations extraeuropéennes, elle concerne cependant également d'autres registres, comme l'"art populaire", l'“art brut", l'“art des fous" ou encore l'“art préhistorique" ". Nous pourrions ajouter qu'elle se pose bien au-delà de ce qui semble demeurer le propre de l'anthropologie de l'art, les primitifs dans toutes leurs acceptions. C'est en la transposant au monde des collections d'art ou de beaux-arts que la notion nous paraît également opérante. Il ne faut pas en rester au seul parcours du cabinet de curiosité au musée du Louvre en passant par le musée d'ethnographie, bien d'autres biographies d'objets montrent ces processus de requalification incessants.

De même le substrat symbolique inscrit dans la notion de butin touche les œuvres d'art classiques ; évident dans les objets pris par fait de guerre au roi d'Abomey Béhanzin, le terme réapparaît sous la plume de Paul Rivet et GeorgesHenri Rivière pour désigner, au retour de l'expédition Dakar-Djibouti «la récolte de 3500 objets ethnographiques, notation de 30 langues ou dialectes [...], formation d'une importante collection de peintures abyssines [...] formation d'une collection de plus de 300 manuscrits et amulettes éthiopiens destinés à la Bibliothèque Nationale, rassemblement pour le Muséum d'une collection zoologique [...], prise de 6000 clichés photographiques, et de 200 enregistrements sonores [...], recherches archéologiques, topographiques, anthropologiques, entomologiques, embryologiques, botaniques, etc. " " Tel est le butin rapporté par cette expédition... ${ }^{4}$ " Or il peut désigner les expéditions napoléoniennes en Égypte, en Italie ou en Allemagne...

Finalement aucun objet ne semble échapper au déplacement, la collection et la patrimonialisation étant pas définition transfert d'un usage à un autre et d'une

1. Le 4 juin 2013, sous le titre Les modalités de la collecte : rapt, troc, marché, fouilles, don... et leur impact sur l'objet.

2. « La qualification de I'objet. Leçon d'introduction au séminaire doctoral d'Histoire de I'art appliqué aux collections », Cahiers de l'École du Louvre, n² 2, 2013 :

http://www.ecoledulouvre.fr/revue/numero2/Jarrasse.pdf

3. http://www.quaibranly.fr/fr/enseignement/la-recherche/le-gdri-anthropologie-et-histoiredes-arts.html, consulté le 25 avril 2014.

4. «La Mission ethnographique et linguistique Dakar-Djibouti », Minotaure, n², 1933, pp. 3-5. 
catégorie à l'autre. Thomas McEvilley, dès 1992 dans L'Identité culturelle en crise, livrait ses réflexions à l'occasion d'une exposition d'art africain contemporain :

"Voir un objet ailleurs que là où il a été fait et à un autre endroit que celui pour lequel il a été fait, c'est le voir avec des questions comme : comment et pourquoi est-il arrivé à ce nouvel endroit ? A-t-il été volé par quelque force impériale ? Est-ce que son itinéraire porte la marque de l'exploitation économique ou de la violence ? Les indiens Zuni et quelques peuples non européens ont demandé le retour de leurs objets exposés dans les musées d'art et d'ethnographie occidentaux, parce que ces objets ont des pouvoirs spécifiques qui sont perturbés quand ils sont dans un environnement culturel étranger. Le gouvernement de Grèce demande depuis longtemps le retour des marbres d'Elgin du British Museum, parce que les objets sont liés au lieu de leur réalisation. Il est peu vraisemblable en revanche qu'un pays d'Europe occidentale demande le retour depuis le Japon ou d'ailleurs de, disons, des peintures des vieux maîtres. Ce sont des relations de pouvoir qui gouvernent ces sentiments (comme les autres). La dispersion des objets culturels occidentaux dans des cultures non occidentales est perçue comme une affirmation du pouvoir et de l'influence occidentale, une sorte de mission de prosélytisme, mais le confinement des objets non occidentaux dans les musées occidentaux est vu d'une manière complètement opposée, comme un signe de la soumission de l'autre culture au pouvoir occidental, de l'appropriation de sa richesse et, bien sûr, de son identité par une force étrangères."

Sans doute d'un schématisme trop rigoureux, ce parallèle rappelle non seulement que nous sommes entrés dans une phase de réclamations complexes, un terrain éthique qui n'est pas abordé ici, mais démontre surtout la relativité des points de vue, le poids des modalités de la collecte des objets, et, de ce fait, leur impact sur leur statut à travers leur mise en collection. Mais le déplacement forcé des objets ne s'est pas produit uniquement dans le cadre de l'impérialisme occidental sur le reste du monde, à l'image du sac du Palais d'été en octobre 1863 sous la houlette de lord Elgin et du général Montauban ; c'est un processus interne au double processus symbolique (exposition du butin ou don diplomatique) et économique (marchandisation des objets et des œuvres d'art) qui a animé ces échanges. L'histoire de l'art est ponctuée d'exactions depuis l'Antiquité : les pillages romains en Grèce, la quatrième croisade détournée en conquête de Constantinople $(1204)^{6}$, les spoliations napoléoniennes et nazies n'ont rien à envier à la manière dont se sont constituées les collections d'arts indigènes, dont le rapt du kono chez les Dogons, relaté par Leiris dans L'Afrique fantôme (1934), est devenu un symbole, encore peu mis en lumière. Le cas des arts indigènes demeure crucial, non seulement comme le souligne McEvilley par la disproportion entre les pouvoirs des cultures mises en relation, mais par un jeu subtil d'artification qui transforme les objets. L'objet quotidien (et donc de peu de valeur), l'objet religieux (sans valeur économiquement définissable), la production d'un enfant ou d'un " fou ", document clinique, en un mot les objets non qualifiés comme art par leurs producteurs, une fois entrés en possession du collectionneur (et du savant) reçoivent un nouveau statut et relèvent désormais d'un marché différent... C'est encore un point de conjonction entre art brut et art primitif Cependant, comme l'évoque l'article de Vincent Capt, l'attention et le respect rendus au créateur peuvent venir atténuer ce qu'il y avait à l'origine de négation de la personne de l'artisan-artiste, de quasi déni d'identité.

5. « L'identité de l'Autre. Réflexions d'un Occidental à l'occasion d'une exposition d'art contemporain d'Afrique », L'Identité culturelle en crise. Art et différence à l'époque postmoderne et postcoloniale (1992), trad. franç., Nîmes, Editions Jacqueline Chambon, 1999, p. 77.

6 . On peut s'étonner que la «fondation de I'empire latin d'Orient » ait pu être inscrite aux Commémorations nationales, célébrant le fait que les barons aient « rapportés en Occident des objets précieux et des reliques (celles de la Passion furent à l'origine de la construction de la Sainte-Chapelle) qui ont influencé la dévotion et la peinture du XIII ${ }^{\mathrm{e}}$ siècle, tandis que des contacts intellectuels encourageaient notamment la diffusion de l'aristotélisme. ». Il est vrai que ramener des émaux pour la Pala d'Oro et les chevaux de San Marco fut une forme de contact. La vision partielle et ethnocentrique de I'histoire a encore de beaux jours devant elle. 
Les textes réunis montrent comment le recoursà des démarches anthropologiques peut avoir cet effet fructueux de rendre plus sensible dans la qualification des objets, non seulement le poids de la mise en collection, comme cela a déjà été démontré, mais aussi l'impact des modalités d'acquisition et de transfert. De même, y perçoit-on comment la collection devient elle-même, par-delà l'amour de l'art, objet aux fonctions économiques, sociales et symboliques. La passion qui y préside engendre même des comportements extrêmes comme celui de Botkine faisant fabriquer des émaux géorgiens pour rendre sa collection inégalable, et une figure comme le chevalier Durand laisse entrevoir une forme de collection des collections. 